\title{
Multimedia Data in Cloud Storage System using DE Duplication Verification
}

\author{
Kotte Ajay \\ M-Tech Dept. of CSE \\ Gokaraju Rangaraju \\ Institute of Engineering and Technology, \\ Hyderabad, TS
}

\author{
G. N. Beena Bethel \\ Associate Professor \\ Dept. of CSE Gokaraju \\ Rangaraju Institute of Engineering and \\ Technology, \\ Hyderabad, TS
}

\begin{abstract}
A Hybrid cloud is a concrescence of public plus private clouds bound together by either standardized or proprietorship technology that changes information plus diligence immovableness. Proposed system aiming to expeditiously resolving yequandary from deduplication on differential favors in remote location computing. A hybrid remote location structure lying of a populace remote location plus a person removed location plus ye information owners simply source their information storage by using world cloud while ye information operation is managed in private cloud. To build information management measurability in cloud computing, de-duplication has been an identical well-kenned technique recently is use. Deduplication dilutes your bandwidth requirements, hastens the data transfers, and it keeps your cloud storage needs to a minimum. Proposed system demonstrates several nascent deduplication formulas fortifying approved duplicate assure inside hybrid remote location structure. To hold ye privacy of information ye convergent encoding proficiency holds made up used to encrypt ye information afore source. Approved deduplication system support differential sanction duplicate check. As a proof of conception, a prototype is implemented in approved duplicate check scheme and conduct test bed experiments utilizing prototype, approved duplicate check scheme incurs minimal overhead compared to mundane operations.
\end{abstract}

\section{Keywords}

De-duplication, Proof of possession, Convergent encoding, Key Management.

\section{INTRODUCTION}

To make information management scalable in cloud computing, deduction has been a well-kenned proficiency plus has magnetized more plus more care recently. Information deduplication is a specified information compaction method for abnegating duplicate replicas of reiterating information in recollection. The method is utilized to ameliorate recollection utilization plus can withal be habituated to network information transports to dilute ye number of bytes that mustiness be sent. In lieu of keeping numerous information copies with yesimilar content, deduplicationexcretes superfluous information by holding only solitary physical copy plus referring further tautological information to tautological imitate. Deduplication can carry lay at yedata records level or yechunk level. For data records level deduplication, infotech rejects reiterate facsimiles from yelike data records. Deduplication can adscititiously choose home astatine yechunk level, which excretes double chunks from information that occur in non-identical data records.

Albeit information deduplication contributes an cornucopia of benefits, aegis plus secrecy pertains stand up while utilizer's sensitive information are sore to some insider plus foreigner comings .Traditional encoding, while supplying information concealment, is incompatible with information deduplication. Concretely, natural encoding desires different utilizer to cipher their information on their have keys. Thus, very information replicas of different utilizer will lead to unlike cipher texts, constructing deduplication infeasible. Convergent encryption has been suggested to enforce information prudence while constructing deduplication executable. InfoTech cipher text/mundane text an information copy with a confluent key, which is obtained through computing the cryptanalytic hash measure from yemessage fromyeinformationimitate.Afterward key propagation plusinformationencoding, utilizer'shold yekey valuesplussend outyeciphertext to yeremote location. Afterwards ye encryption process is deterministic plus is derived from the information content, identical 1 information copies will cause the Sami merging key plus hence the same cipher text. To avert wildcat access, a insure proof of possession protocol is nevertheless demanded to supply the proof that the utilizer indeed owns yeLapp data file whenever a double is detected. Afterward yeproofread, subsequent utilizer 'son yeLapp data file volition be supplied an arrow of yewaiter less wanting to transfer yelike data file. A utilizer can download yecipher text records with yearrow of yehost, which can alone be decoded by yerepresenting information owners with their focused keys. Hence, convergent encryption approves ye remote localization to perform deduplication on yeciphertextsplus ye proof of ownership obviates ye unauthorized utilizer to get at yedata files.

\section{RELATED WORK}

Hybrid cloud can be built utilizing any technology it changes granting to unlike traffickers. Key components in many of the situations, implementation of the hybrid cloud has a comptroller that will hold chase of all placements of private and public clouds, IP address, servers and other resources that can run systems efficiently.

\subsection{Existing System}

Data deduplication be solitary of consequential information compression techniques for abnegating duplicate replicas of reiterating information, and has been widely utilized in cloud recollection to reduce the sum of recollection space plus preserve bandwidth. To forfendye confidentiality of sensitive information while strengthening deduction, Cloud computing provide ostensibly illimitable "virtualized" resources to users as accommodations throughout the completely Internet, as obnubilating platform and implementation details. Today's cloud accommodation providers offer some highly useable storage plus massively parallel calculating resources at relatively low costs. As remote location computing becomes dominating, an incrementing count from information makes 
up renovated in yeremote location plus shared by utilizer's with designated favors, which decide the approach redresses of yememory information.

\section{Disadvantages of Existing System:}

- One critical challenge of cloud recollection accommodations is the management of ye everincrementing volume of information.

\subsection{Proposed System}

Hybrid Cloud can be built utilizing any technology it changes granting to unlike vendors. Key constituents in lots of the situations, implementation of the hybrid cloud has a comptroller that will hold track of everyone perspectives of secret plus public clouds, IP address, server's plusearlyresources that can run systems efficiently.

\section{Some of the key components include}

- Orchestration manager plus cloud purveying for storage, populace cloud imaginations which lets in virtual machines plus networks, the private and public clouds, which are not compulsorily compatible or identical.

- Synchronization element and Data transfer expeditiously supersede information among private plus public clouds.

- Changing configurations of storage, network and some early resources are constituting crossed by configuration monitor.[1]

In the Fig 1, the simplest view of hybrid cloud is approved for, a single off-premises public cloud plus on-premises secret cloud is amongst ye Enterprise Datacenter is shown plus public cloud demonstrates the safe sodality to store information on to the cloud is denoted by the arrow:

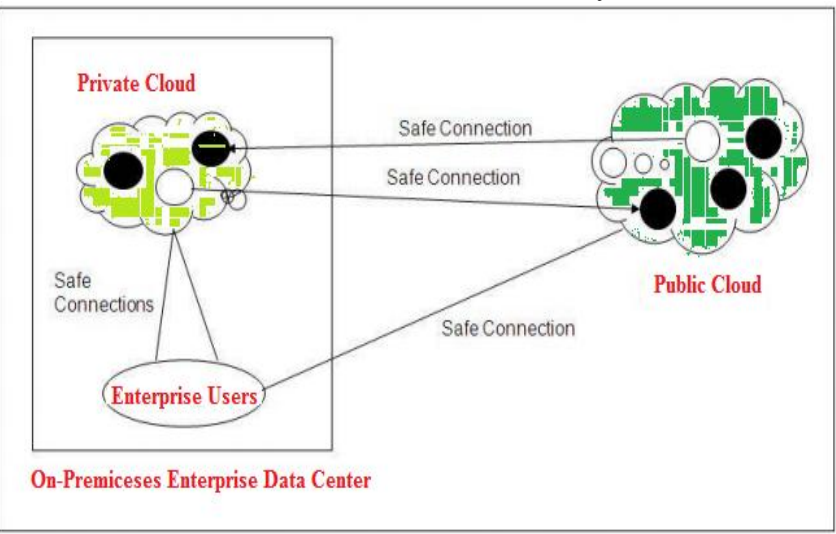

Fig 1: Hybrid Cloud Environment

The ebony bands show dynamic virtual host images and white circles shows virtual server images which have been migrated by applying good connections. The arrows designate that the direction of migration. Utilizing safe connections initiative utilizer are associated to ye clouds, which can be ensure HTTP browsers or virtual private networks (VPNs) .A hybrid cloud could adscititiously can consist of multiple public or/and private clouds. [3]

Information de-duplication has lots patterns. Generally, there is no one best way to enforce information de-duplication across a total an establishment. Instead, to maximize the gains, systems may spread more than one de-duplication strategy. It is very necessity to realize the backup plus backup challenges, when culling de-duplication as a solution.
We have inserted hybrid cloud computer architecture in our aimed deduplicationscheme. The private keys for prerogative will not be furnished to utilizer immediately, which will be held plush plus led by ye private cloud server rather. In this fashion, ye utilizer cannot donation these private keys of favors in this suggested structure, which betokens that it can evade ye privilege key distributing amongst utilizer in the over straight structure. To get a data file keys, ye utilizer ineluctably to ship a call for to yeindividual remote location waiter. Ye suspicion from such building can be described as comes. To do the duplicate assure for some data file, the utilizer wants to get yedata file keysonyeindividualremote location waiter. The Individual outside location waitron will adscititiously assure yeutilizer'sindividuality afore publishing ye representing data file keys to ye utilizer. The approved double assure as such information data file bum be did throughye utilizer on yepopulateremote location afore transmitting this information records. Predicated on yeanswers of double assure, ye utilizer either uploads this data file or runs PoW.

\section{IMPLEMENTATION}

Afore affording our construction of yededuplicationscheme, we decide a binary cognation $\mathrm{R}=\mathrm{f}\left(\left(\mathrm{p}, \mathrm{p}^{\prime}\right) \mathrm{g}\right.$ because comes. Given 2 privileges $p$ plus $p^{\prime}$, we verbally show that $p$ corresponds $\mathrm{p}^{\prime}$ if plus only if $\mathrm{R}\left(\mathrm{p}, \mathrm{p}^{\prime}\right)=1$.

\subsection{System Setup}

An identification protocol ${ }_{-}=$(Proof, Verify) is supple mentally determined, where Proof plus trust appoint ye validation plus check algorithm severally. Moreover, for apiece one utilizer $U$ subsists suspected to have a whodunit key skU to execute ye identification with waiters. Postulate that utilizer $U$ features yefavor adjust PU. It adscititiously formats a PoWset of rules POW for yedata records ownership proof. The individual cloud server will assure a table which shops each utilizer public information pulps its representing privilege set PU.

\subsection{File Uploading}

Suppose that information proprietor requires to transfer plus apportion a data records $\mathrm{F}$ on user's whose privilege belongs toye set $\mathrm{PF}=$ fpjg. The information owner demands act with yesecret remote location afore acting duplicate check with ye S-CSP. Information owner does recognition to endeavor out infoteches individuality on secret tokensskU. If it is communicated, yesecreteremote locationwaitertestamentget yerepresenting favors PU of ye utilizer of its recollection table list. The utilizer estimates plus ships ye information data records tag $\phi \mathrm{F}=$ TagGen $(\mathrm{F})$ to yesecreteremote location waiter, who will return $\mathrm{f} \phi^{\prime} \mathrm{F} ; \mathrm{p}_{-}=\operatorname{TagGen}\left(\phi \mathrm{F}, \mathrm{kp} \_\right) \mathrm{g}$ back to the utilizer for total $\mathrm{p}_{-}$gratifying $\mathrm{R}\left(\mathrm{p}, \mathrm{p}_{-}\right)=1$ plus $\mathrm{p} 2 \mathrm{PU}$. Then, the utilizer will act plus ship ye file token $f \phi^{\prime} F ; p_{-} g$ to y S-CSP.

If a double data is detected by ye S-CSP, ye utilizer perpetuates proof of ownership of this data file with ye SCSP. If the cogent evidence is authorized, ye utilizer will be allotted a pointer, which approves him to access ye file.

Otherwise, if no duplicate is found, the utilizer computes the encrypted file $\mathrm{CF}=\operatorname{EncCE}(\mathrm{kF}, \mathrm{F})$ with ye convergent key $\mathrm{kF}$ $=\operatorname{KeyGenCE}(\mathrm{F})$ plus uploads $\left(\mathrm{CF}, \mathrm{f} \phi^{\prime} \mathrm{F} ; \mathrm{p} \mathrm{g}\right)$ to ye cloud host. Ye convergent key $\mathrm{kF}$ is stored by the utilizer locally.

\subsection{File Retrieving}

Conjecture a utilizer requires to getting a data records F. It beginning sends out ancall for plusyedata records name to ye 
S-CSP. Upon arriving ye petition plus information file designation, the S-CSP will assure whether ye utilizer is worthy to download F. If failed, the S-CSP sends back a terminate signal to the utilizer to denote yedata getting from network loser. Differently, ye S-CSP affords the representing ciphertextCF .on experiencing yeciphered information from ye S-CSP, the utilizer utilizes ye key kFmemorytopically to recuperate ye pristine $€$ file $F$.

\section{EXPERIMENTAL WORK}
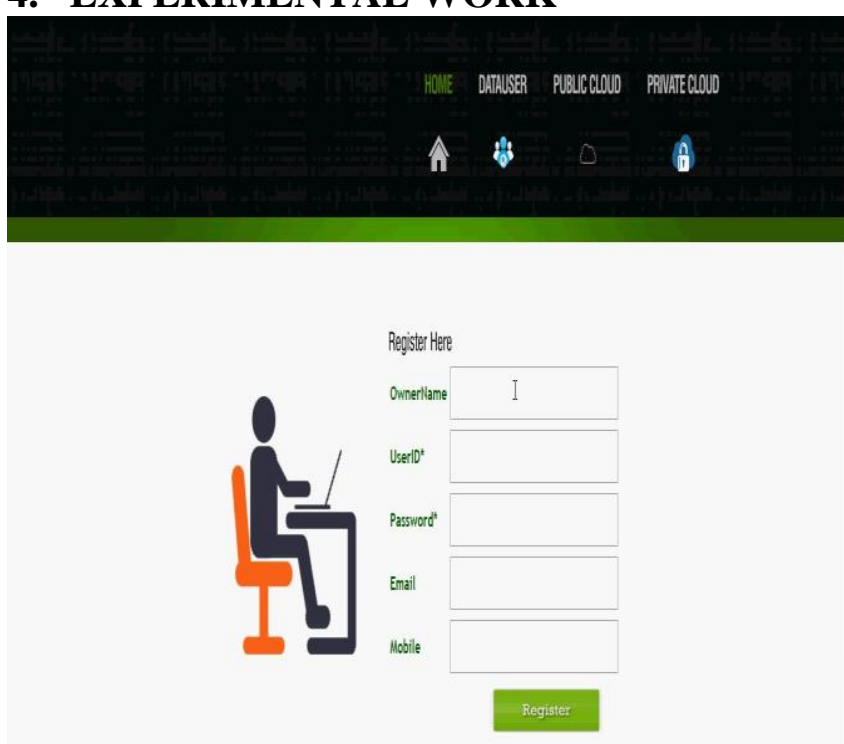

Fig: 2 New Account Opening
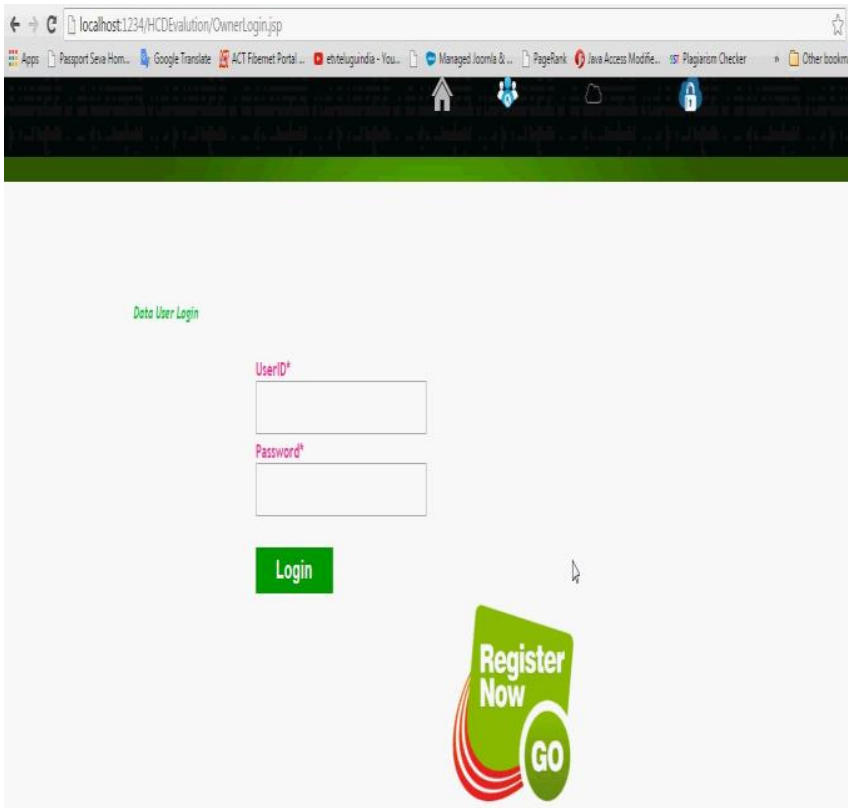

Fig: 3 Secure Login
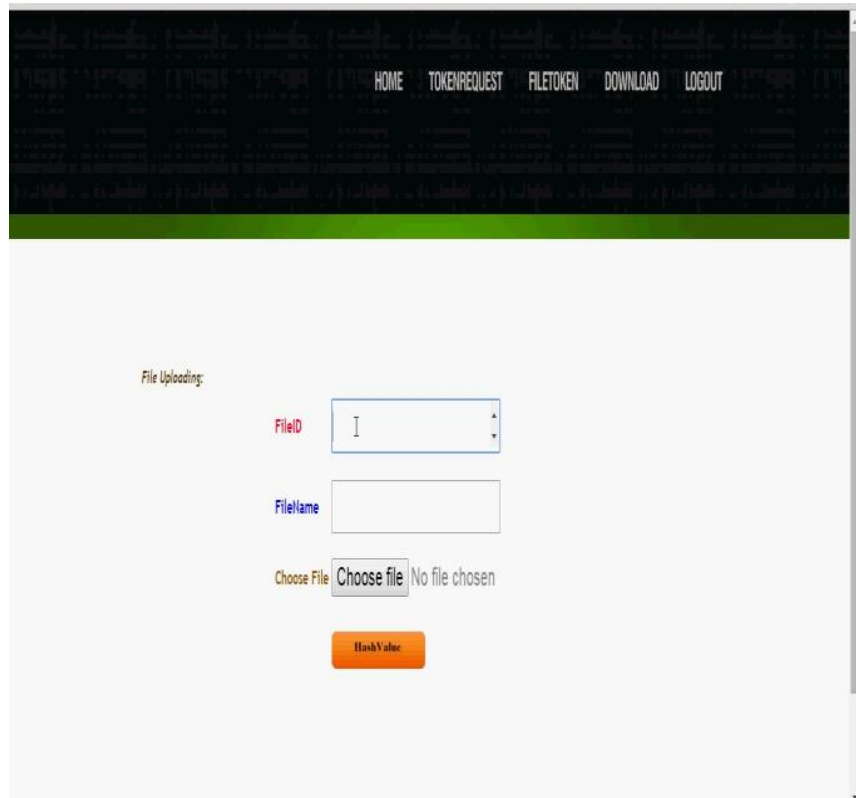

Fig: 4 Data upload

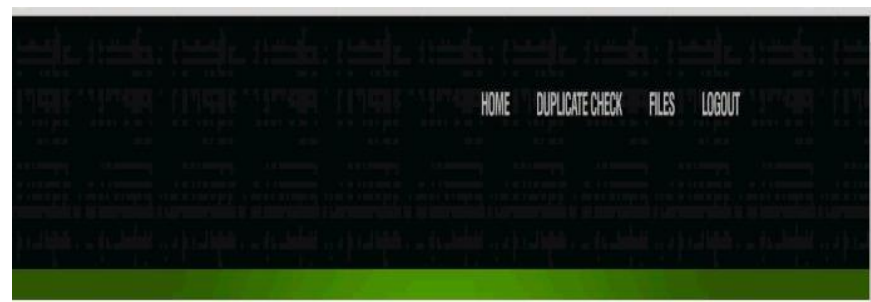

WeCOU: TO PUACCLOW 


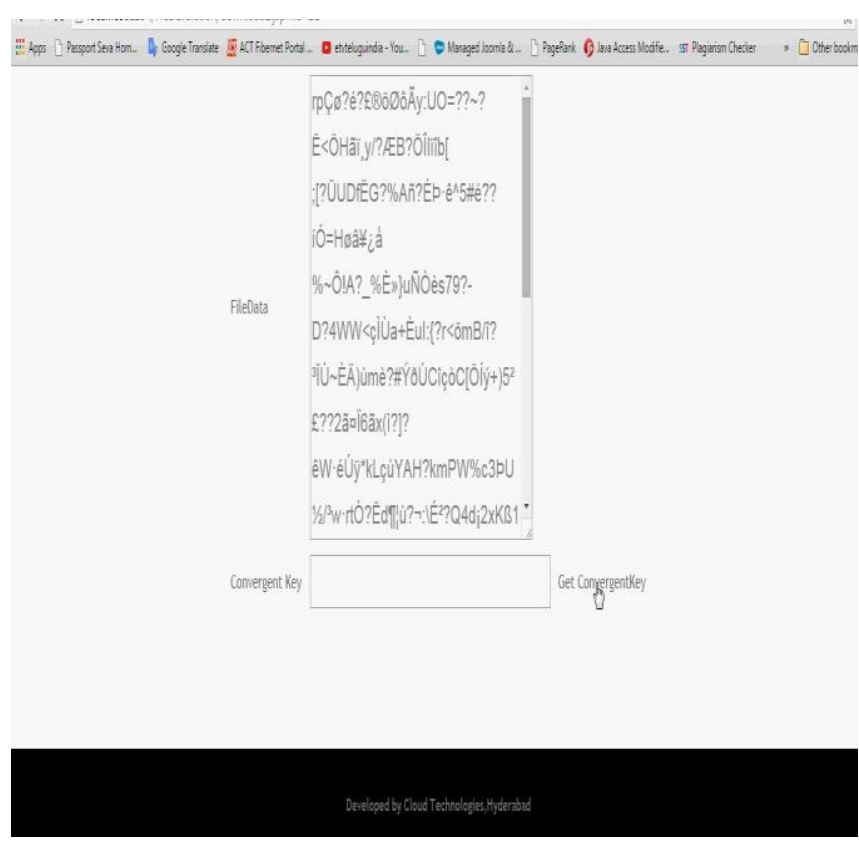

Fig: 6 Encryption
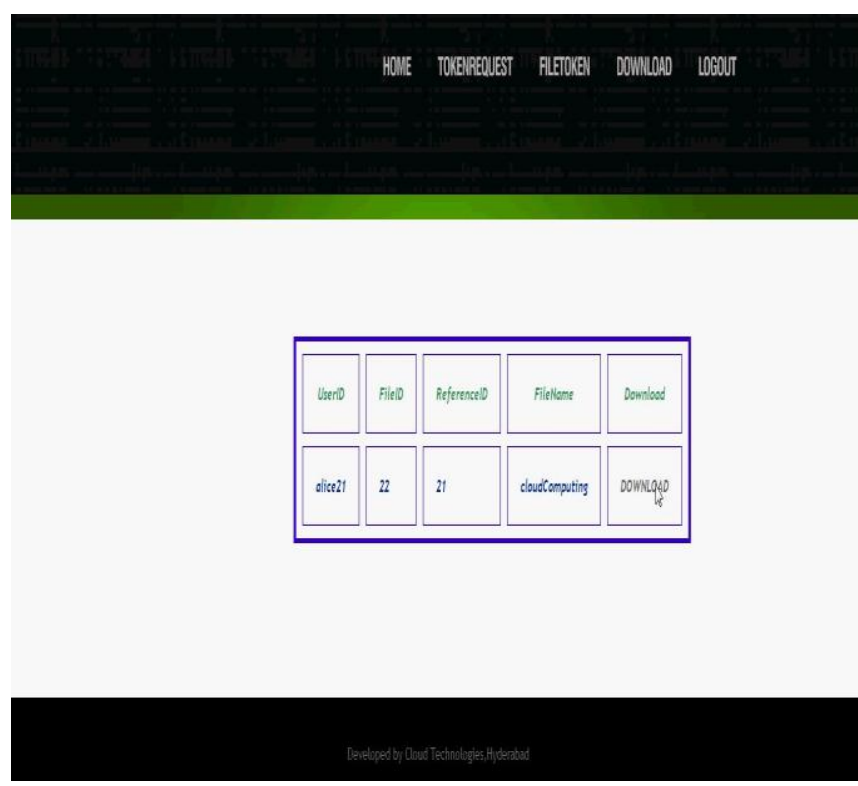

Fig: 7 File Download

\section{CONCLUSION}

The cerebration of approved information deduplicationissuggested to ascertain the information security through counting disparity gains of clients in yeduplicate replica check. The presentation of aelite incipient deduplicationgrowths fortifying approved duplicate reengender in hybrid cloud architecture, in that ye duplicate assure tokens of documents are caused via ye private remote locationwaiterholding secrete keys. Security check presents that ye methods are assure regarding insider plus foreigner attacks elaborated in ye suggested security model. As an issue verification of conception ion, the developed model of the proposed approved duplicate copy check method and tested the model. That showed the approved duplicate copy check method feel minimum smash equating convergent encryption and data transfer.

\section{REFERENCES}

[1] Bugiel, Sven, et al. "Twin clouds: Secure cloud computing with low latency." Communications and Multimedia Security. Springer Berlin Heidelberg, 2011

[2] Anderson, Paul, and Le Zhang. "Fast and Secure Laptop Backups with Encrypted De-duplication." LISA. 2010.

[3] Bellare, Mihir, SriramKeelveedhi, and Thomas Ristenpart. "DupLESS: server-aided encryption for deduplicated storage." Proceedings of the 22nd USENIX conference on Security.USENIX Association, 2013.

[4] Bellare, Mihir, SriramKeelveedhi, and Thomas Ristenpart. "Message-locked encryption and secure deduplication."Advances in Cryptology-EUROCRYPT 2013.Springer Berlin Heidelberg, 2013.296-312.

[5] Bellare, Mihir, ChanathipNamprempre, and Gregory Neven. "Security proofs for identity-based identification and signature schemes." Journal of Cryptology 22.1 (2009): 1-61.

[6] M. Bellare, S. Keelveedhi, and T. Ristenpart.Dupless: Serveraided encryption for deduplicated storage. In USENIX Security Symposium, 2013.

[7] K. Zhang, X. Zhou, Y. Chen, X.Wang, and Y. Ruan.Sedic: privacyaware data intensive computing on hybrid clouds. In Proceedings of the 18th ACM conference on Computer and communications security, CCS'11, pages 515-526, New York, NY, USA, 2011. ACM.

[8] S. Halevi, D. Harnik, B. Pinkas, and A. ShulmanPeleg.Proofs of ownership in remote storage systems. In Y. Chen, G. Danezis, and V. Shmatikov, editors, ACM Conference on Computer and Communications Security, pages 491-500. ACM, 2011. 